\title{
Investigation and Development of IoT Based Solutions for Implementation the Cloud Technologies in the Energy Systems Control
}

\author{
P. Yakimov, A. Iovev
}

Key Words: Cloud computing; infrastructures for industrial informatics; IoT; sensor networks; Smart grids.

\begin{abstract}
The paper considers the possibilities for implementation the Cloud technologies in the energy systems control and the expected benefits from this. The trends in the electric energy systems development and the challenges in front of the control are summarized. It is obvious that the introduction of information and communications technologies is unavoidable in order to achieve the main goals - effective, reliable, sustainable and safe operation of the energy system. A possible scenario is the development of a modern metering infrastructure based on smart sensor networks. Development and implementation of Internet of Things oriented solutions is expected to help the introduction of Cloud computing in the industrial and home energy management systems. Two models of IoT based solutions are explained. They consider the approaches for Internet connectivity of modern and legacy measurement and control equipment. Experimental results are given.
\end{abstract}

\section{Introduction}

The European Commission has accepted the Renewable Energy Directive, which main goals are to achieve a $20 \%$ reduction in greenhouse gas emissions, $20 \%$ renewable energy consumption, and a $20 \%$ reduction in energy consumption by 2020 (compared to the levels in 1990). This is determined by the requirements for energy efficiency and environment protection. The modern trends are limitation the usage of coal and oil derivatives as energy sources, and expanding the generation from renewable energy sources (RES) like water, wind and solar radiation. Another step in this direction is the increase of the electric vehicles. A significant impact on the power systems operation is given by the liberalization of the energy market. These major challenges determine a new phase in the energy systems development emphasizing on the modernization of the grid. This modernization is spread over all parts of the energy chain -generation, transmission, distribution and consumption [1]. Power systems follow the development of computer systems and from hierarchical and centralized they become distributed. In every part of this complex system there is a transformation which changes its character. Along with the traditional bulk generation there are the wind and solar power plants which production depends on the environmental conditions, hence it is difficult to predict it. Also it is difficult to predict the demands requested by the electric vehicles charging stations and by the industrial and home consumers. Thus the substations bridging the transmission lines and the distribution domain need additional smartness in order to be able to connect consumers to the energy producers. The transformers there decrease the voltage levels from high transmission values to lower distribution voltage values. In the distribution system the voltage is stepped down again to the appropriate values for the customers [2]. Because of that substations are basic points in the power grid and the safety and sustainable functioning of the whole system depends on their proper operation. The fast and correct switching in the substations have to ensure uninterruptible power supply for the consumers with minimum faults in the system. This means that the grid protection and power control rely on their correct and fast functioning. There are installed advanced metering, intelligence, and automation in order to monitor and manage more effectively the local system aiming at limiting the faults propagation and their impact on the rest of the grid by switching off the proper circuit breaker.

So the modernization of the power system is facing the challenges and the key to overcome them is in developing Smart grids. The model of a Smart grid is the integration of the information and communication technologies in the power system. Hence along with the traditional performers on the energy field - generation, transmission, distribution and users, the new ones - markets, operators and service providers are involved [3]. Weather forecasts and demands models profiling begin to play important roles in the power systems control. Thus there is a need of big data storage and processing. In general in Smart grids there is a bi-directional communication between the participants in the energy chain along with the power flow. The highlights of Smart grids are monitoring, protection, automation, optimization, integration and security of the power flow from utility generators to the end consumers.

This paper is organized as follows: section 2 outlines some advantages of IoT and the cloud computing in order to motivate their implementation in the network-based control in the power system. The proposed infrastructure models, the hardware and software design are described in section 3 . The conclusions and the future work are presented in the last section.

\section{IoT and Cloud Computing Essentials}

Internet of Things has originated at the Massachusetts Institute of Technology (MIT) in the beginning of the 21 st century like many other innovative concepts, especially in the technical field [4]. Its main goal is to support the development of smart environments for application in 
different areas of the industry and social life like e-government, e-learning, e-health, e-business, smart homes, smart cities and etc. This technological jump has become possible as a result of the strong progress of electronics, computing and telecommunications. The term "thing" is used for people, machines, devices, sensors and data [5], or the essence of the IoT concept means their integration to Internet.

The implementation of two important achievements IPv6 and Cloud computing, has given a significant support to the Internet of Things. IPv6 allows the communicability, giving an address to billions of devices. Additionally, it eases the management of the networks because of the capabilities for auto configuration and offers improved security features as well. Cloud computing is already a building block of the Internet and the Internet of Things is expected that will be the biggest user of Cloud. Cloud environment is also able to provide the services for analysis of the data streams in order to support ensuring the information to the end consumer in an optimal form. Recently cloud computing is being more widely used in the government, education, business, healthcare, and etc., but it is relatively new as application in SCADA systems. The main components of these systems are Remote telemetry units
(RTUs), Programmable logic controllers (PLCs), a supervisory computer-based system, databases, Human-Machine Interfaces (HMIs) and etc. The cloud computing allows the virtualization of some of the components of the SCADA systems except the RTUs, which are physical devices and are connected to the objects. The functions of the others can be distributed between various machines where heavy software algorithms can be run using the enormous computing resources of the cloud. This is especially valid for the supervisory computer-based systems, databases and HMIs. The cloud computing will enable the processing of big data which is needed in weather forecasts analysis and consumers' demands profiling. Also it will support the control functions using virtualized PLCs. The inputs and the outputs of the controller are connected to the physical object but the control software is running on a virtual machine in the cloud using a big computing capability and heavy software packets like MATLAB and etc., which is impossible in the traditional practice.

The Internet of Things architecture is layered as it is shown in figure 1. It is intended to abstract and automate the integration of objects, and to provide smart services. The model can be divided into three layers [6], respectively perception, transportation, and application.

\begin{tabular}{|l|l|}
\hline \multirow{2}{*}{$\begin{array}{l}\text { Application } \\
\text { layer }\end{array}$} & $\begin{array}{l}\text { Industrial } \\
\text { monitoring, smart home }\end{array}$ \\
\hline $\begin{array}{l}\text { Cloud computing platform, } \\
\text { middleware technology }\end{array}$ \\
\hline $\begin{array}{l}\text { Transportation } \\
\text { layer }\end{array}$ & $\begin{array}{l}\text { Networking capabilities - } \\
\text { Local area network, Internet, } \\
\text { WiFi }\end{array}$ \\
\hline $\begin{array}{l}\text { Perception } \\
\text { layer }\end{array}$ & $\begin{array}{l}\text { Field devices - Sensors, } \\
\text { actuators }\end{array}$ \\
\hline
\end{tabular}

Figure 1. IoT systems architecture model

The perception layer is responsible for the front-end in order to recognize and control the field devices, and also to collect the data provided by them. It contains transducers (sensors and actuators), signal conditioning and data conversion blocks, and an application processor. The ana$\log$ output signals of the sensors are conditioned and scaled (amplified), then converted to a digital format by an $\mathrm{A} / \mathrm{D}$ converter. The digitized sensor signals are processed by a microprocessor using a digital application control algorithm. The transportation layer ensures the communicability of the elements of the perception layer giving them an ubiquitous network access. The measured or calculated parameters are transferred to the host or monitoring system in a network by means of network communication protocols. The appli- cation layer is intended for smart services provision, Human-Machine Interfaces (HMIs) machine-to-machine (M2M) communications and etc.

\section{Implementation of IoT Based Solutions for Power System Measurement and Control}

IoT based devices can be fixed or mobile. The fixed ones (being in fixed locations) might use cable or wireless Internet connection. The mobile devices can be wirelessly connected to the Internet (e.g., by mobile phone). According to the networking capabilities the IoT based devices 
can be considered as ones working in active mode and others which operate in passive mode. When the thing itself is connected to the Internet, it is in active mode and is able to communicate in real-time. By now, there are many devices and sensors which are used in the power system monitoring and control, but they are not connected directly to the Internet. These devices could be connected as things to the Internet in a passive mode through the concentrated devices (gateways or mediators) which support sub-networks and are connected to the Internet [10]. In the passive mode, a thing is not connected to the Internet, but can be uniquely identified through the gateway which maintains the network of smart units.

\section{A. IoT Based Power Measurement Transducer Working in Active Mode}

The smart power measurement transducer is developed following the architecture model depicted in figure 1. The block diagram is shown in figure 2 and figure 3 presents the view of the remote interface.

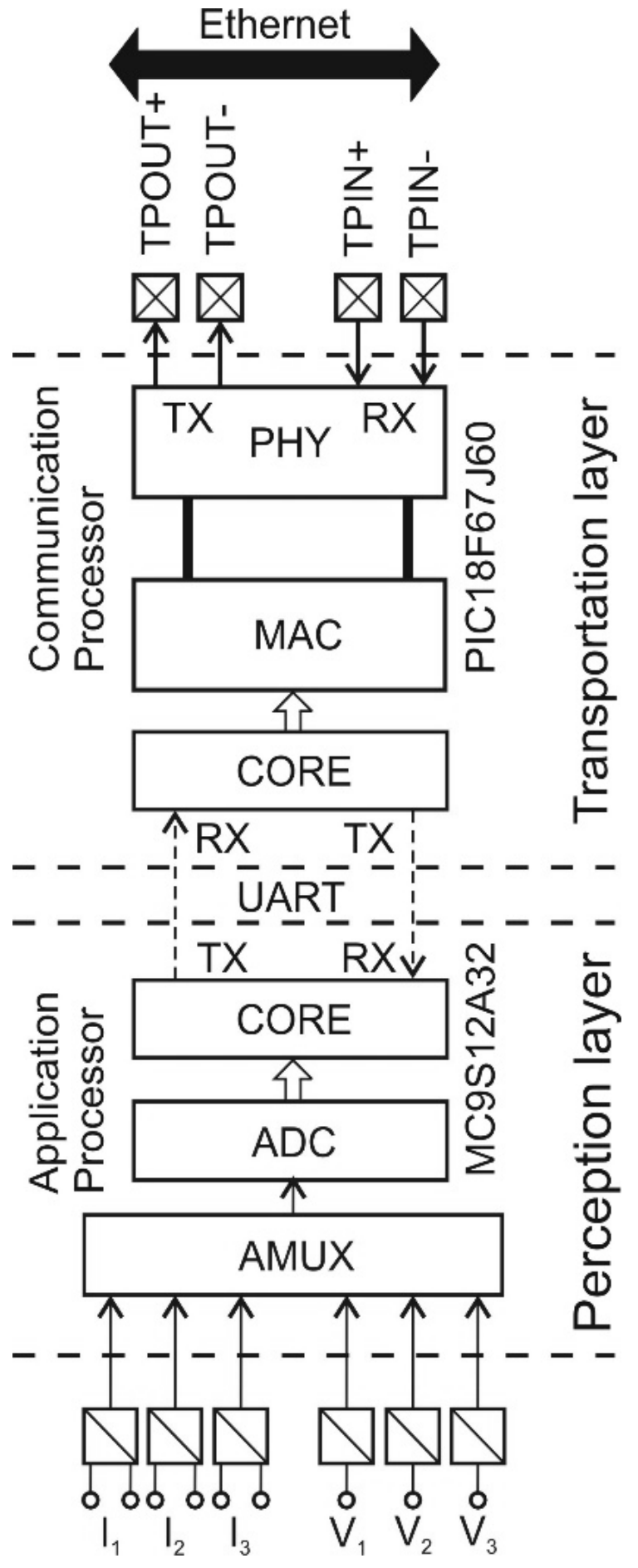

Figure 2. Smart transducer block diagram 
Modern industrial automation and informatics integrates Internet based communication technology which allows direct access to a particular device via the Internet or Intranet. For these purposes it is reasonable to use already existing standards like IP-based communications, standard Web browsers and generally used program languages such as HTML or JavaScript. This allows access by any fixed or wearable device without additional software installation.
The structure of the transducer is divided into two parts, namely Application processor and Communication processor. Every part covers one layer of the IoT architecture model. The parts are based on standard microcontrollers and the data transfer between them is serial using UART. This is a further development of a universal power transducer [7]. The Application processor deals with the input signals and it is connected to the power system by measuring transformers with ratios $\mathrm{kV}$ and $\mathrm{kI}$.

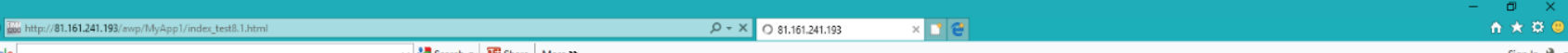

\begin{tabular}{|c|c|c|c|c|c|}
\hline \multicolumn{6}{|c|}{ TRANSDUCER address $=43$} \\
\hline $\mathrm{V} 1=\mathbf{5 7 . 8} \mathrm{V}$ & fi_V1 $=\mathbf{0}$ & $\mathrm{I} 1=\mathbf{1 . 0 0 1 \mathrm { A }}$ & fi_Il $=\mathbf{0}$ & $P=173.6 \mathrm{~W}$ & $\mathrm{f}=\mathbf{5 0 . 0 2} \mathbf{H z}$ \\
\hline $\mathrm{V} 2=\mathbf{5 7 . 6} \mathrm{V}$ & fi_V2 $=\mathbf{2 4 1}$ & $\mathrm{I} 2=1 \mathrm{~A}$ & fi_I $2=\mathbf{2 4 1 . 1}$ & $\mathrm{Q}=0.8 \mathrm{VAr}$ & \\
\hline $\mathrm{V} 3=\mathbf{5 7 . 6} \mathrm{V}$ & fi_V3 $=\mathbf{1 2 1 . 1}$ & $\mathrm{I} 3=1 \mathrm{~A}$ & fi_ $\mathrm{I} 3=\mathbf{1 2 0 . 6}$ & $S=173.6019 \mathrm{VA}$ & \\
\hline \multicolumn{2}{|l|}{$\mathrm{kV}=\mathbf{1 1 0 0}$} & \multicolumn{2}{|l|}{$\mathrm{kI}=\mathbf{1 0 0}$} & $\cos \_i=\mathbf{0 . 9 9 9 9 8 9 4}$ & \\
\hline
\end{tabular}

Figure 3. Remote HMI for data access from the IoT based measurement transducer

The transducer measures the main quantities of the threephase power grid - voltage, current, frequency and phase angles, and calculates the derivatives - apparent, active and reactive power, and power factor. The input signals have rated values respectively $57.7 \mathrm{~V}$ and $5 \mathrm{~A}$ which are standard for the measurement and control systems in the objects of the power grid - power plants, substations and etc. The transducer has the flexibility that after slight adjustments its rated input values can be changed. Thus the voltage dividers can be set for $230 \mathrm{~V}$ and the transducer will be able for direct connection in industrial and home automated systems for energy consumption monitoring. The communication processor is intended to extend the networking capabilities and it supports the remote client interface using IP communications. The processor is fully compatible with 10/100/1000Base-T Networks. Also it supports the HTML page containing the user interface (HMI). The implementation of IP-based communications capabilities gives the transducer possibilities for inclusion in Internet of Things networks.

\section{B. Sensor Networks Development as IoT Based Solutions Working in Passive Mode}

Currently there are a lot of devices and sensors which are used for monitoring and control in the power system, which are not able to be connected to the Internet. Such devices could be connected as things to the Internet of Things in a passive mode through gateways. Gateways interconnect networks by converting the protocols of each network to that used by the other. Thus they make possible the inclusion of legacy equipment which has its original interface protocol to a modern infrastructure. In such cases the gateway bridges the custom developed protocol and a standard one. To integrate/connect and migrate devices, attached to different networks by the use of gateways is a well proven concept, which is applied to transform protocols as well as syntax of data [8]. For the development of sensor networks with extended capabilities for Internet communications it is required to choose the proper gateway. A possible solution is to use a programmable logic controller (PLC) as it is shown in figure 4. The HMI can be viewed in figure 5. PLCs are widely used in the industrial automation and in the process control because they are rugged, reliable and flexible. In the substations of the power grid the PLCs can read the states of relay inputs (contacts) and control relay outputs, e.g. circuit breakers.

Along with the control functions the PLCs have enough communications capabilities to operate as a gateway which can be used to maintain the smart transducers network.

The universal transducers in the sensors network are similar to the described above one. Their communication interface is RS-485 and a custom protocol is used. PLC 1 performs the function of the gateway and it transforms the custom protocol to standard Profibus. For this purpose is used the PLC of Siemens Simatic S7-200 CPU 226, extended with analog module type EM235 and Profibus-DP slave module EM277. Along with the sensors network maintaining the PLC reads four relay inputs and controls four relay outputs, accepts two analog signals from voltage and frequency transducers with analog outputs, and communicates with the upper level. The networking functions are 


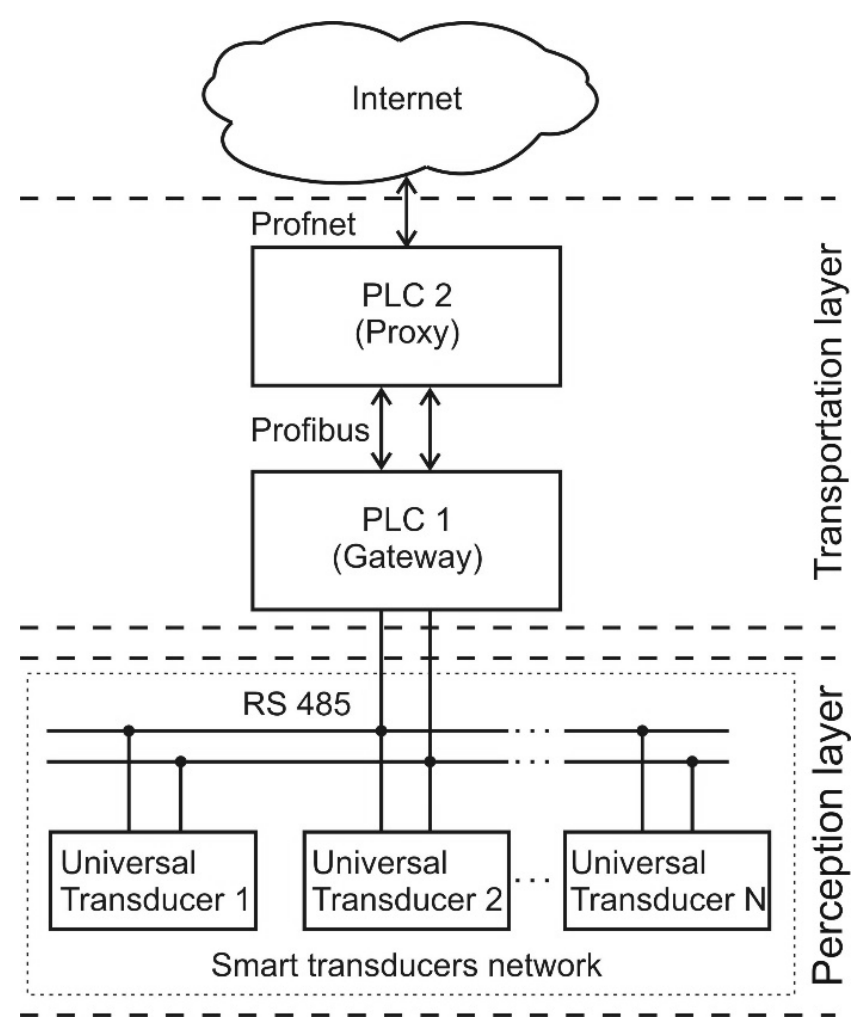

Figure 4. Smart transducers network as IoT solution working in passive mode

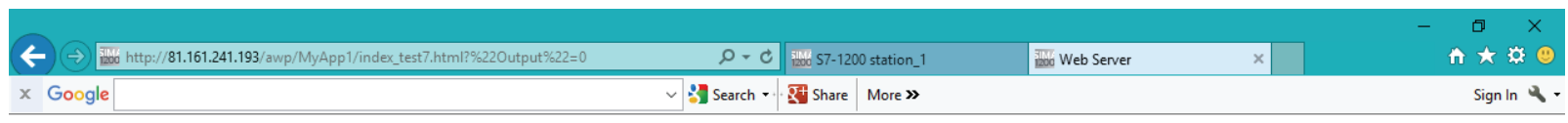

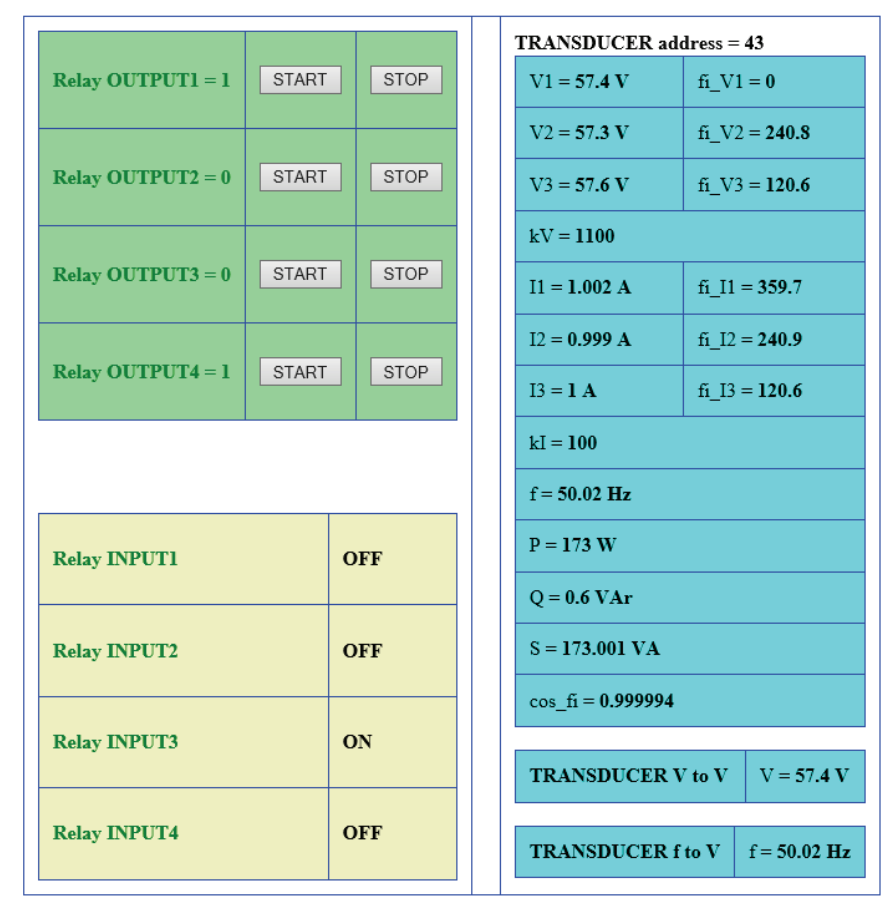

Figure 5. A look of the distant interface for a substation monitoring 
performed by the PLC 2 which works as a proxy for the transducers network. It supports the HTML document which is accessible by the remote clients. The PLC of Siemens Simatic S7-1200 CPU 1214C extended with Profibus-DP master module CM1243-5 is chosen for this application. The proposed infrastructure is suitable for distant monitoring and automation of a substation or another particular object of the power system. The remote monitoring and control of field devices directly from the network has been significantly positively impacted by the growth of Ethernet-based industrial networks such as Profinet and Ethercat.

\section{Conclusions}

In this paper the main features of the IoT concept and Cloud computing are considered. The expected benefits from their implementation in the power grids measurement and control are explained. Two models of IoT based solutions working respectively in active and in passive mode are described. The developed remote HMI is accessible by the clients from anywhere in anytime using Standard Web browsers. Laboratory tests using power system simulator, PLCs and measuring transducers have been conducted. The developed experiments give reliable results for the application of the presented solutions in further investigation of more complex systems for electric power management.

\section{Acknowledgement}

The paper preparation is supported by Project DFNI E02/12 "Investigation of methods and tools for application of cloud technologies in the measurement and control in the power system", funded by Ministry of Education and Science of the Republic of Bulgaria.

\section{References}

1. Giri, J. Proactive Management of the Future Grid. - IEEE Power and Energy Technology Systems Journal, 2, June 2015, No. 2, 43-52. Digital Object Identifier 10.1109/JPETS.2015.2408212. W.K. Chen, Book Style. Belmont, CA: Wadsworth, 1993, 1-15.

2. Fang, S. Misra, G. Xue, and D. Yang. Managing Smart Grid Information in the Cloud: Opportunities, Model and Applications. - IEEE Netw., 26, July 2012, No. 4, 32-38.

3. National Institute of Standards and Technology. NIST Framework and Roadmap for Smart Grid Interoperability Standards, Release 1.0. http://www.nist.gov/public affairs/releases/upload/smartgrid interoperability final.pdf. January 2010.

4. Evans, D. The Internet of Things. How the Next Evolution of the Internet Is Changing Everything. White Paper, Cisco IBSG, 2011. Available: http://www.cisco.com/c/dam/en_us/about/ac79/docs/ innov/IoT_IBSG_0411FINAL.pdf.

5. Turcu, C. and V. Gaitan. An Internet of Things Oriented Approach for Water Utility Monitoring and Control. Advances in Computer Science, Proceedings of the $6^{\text {th }}$ WSEAS European Computing Conference (ECC '12), Prague, Czech Republic, 24-26 September 2012, 175-180, ISSN 978-1-61804-126-5.

6. Jing, Q., A. Vasilakos, J. Wan, J. Lu, and D. Qiu. Security of the Internet of Things: Perspectives and Challenges. - Wireless Networks, 20, 2014, No. 8, 2481-2501.

7. Yakimov, P., S. Ovcharov, N. Tuliev, E. Balkanska, R. Ivanov.
Three Phase Power Transducer for Remote Energy Management System Application. - Annual Journal of Electronics, 4, 2010, No. 2, 31-34, ISSN: 1313-1842.

8. Delsing, J. et. al. Migration of Industrial Process Control Systems into Service Oriented Architecture. 38th Annual Conference on IEEE Industrial Electronics Society, IECON 2012, 25-28 October 2012, Montreal, Canada, 5786-5792, doi: 10.1109/ IECON.2012.6389039.

\section{Manuscript received on 20.11.2017}

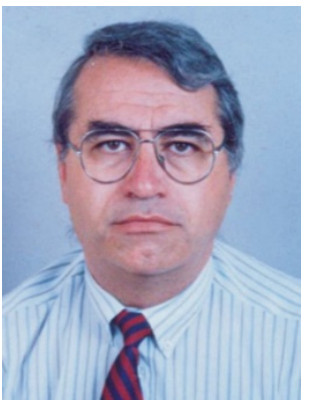

Peter Yakimov was born in Montana, Bulgaria, in 1961. He received his M.S. and Ph.D. degrees in Electronic engineering from the Technical University of Sofia, Bulgaria, in 1984 and in 2003 respectively. He joined the Technical University of Sofia in 1984 as a design engineer at the R@D Division and in 1989 became an Assistant Professor. Since 2007 he has been an Associate Professor. Dr. Yakimov is a co-author of books and manuals in electronic circuits design and an author and coauthor of more than 80 papers in journals and conference proceedings. Currently Dr. Yakimov is a Head of the Department of Electronics at the Technical University of Sofia. His research interests are in the areas of analog, digital and mixed signals circuits design, embedded systems, energy measurements, control systems. He has been a team leader or participant of several science and research projects with local and international funding. Dr. Yakimov is a scientific secretary of the Annual International Scientific Conference "Electronics" (IEEE conference record \#44934). He is an IEEE member, IES and IMS.

Contacts:

Department of Electronics

Faculty of Electronic Engineering and Technologies Technical University of Sofia

8, Kliment Ohridski Blvd., 1000 Sofia, Bulgaria tel: +3592965 3265, mobile: +359893341434 e-mail: pij@tu-sofia.bg

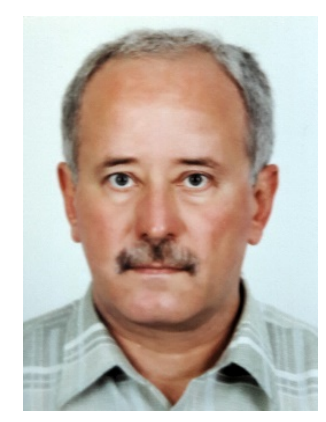

Atanas Iovev was born in Burgas, Bulgaria, in 1955. He received his M.S. degree in Electronic engineering from the Technical University of Sofia, Bulgaria, in 1977. He joined the Technical University of Sofia in 1982 as a design engineer at the $R @ D$ Division and in 1990 became an Assistant Professor. Atanas Iovev is a co-author of books and manuals in electronic circuits design and an author and co-author of more than 30 papers in journals and conference proceedings. His research interests are in the areas of analog, digital and mixed signals circuits design, measurement of nonelectrical quantities, control systems, PLC programming.

Contacts:

Department of Electronics Faculty of Electronic Engineering and Technologies Technical University of Sofia 8, Kliment Ohridski Blvd., 1000 Sofia, Bulgaria tel: +359 2965 3265, mobile: +359895 588815 e-mail:iovev@tu-sofia.bg 